\title{
Atendimento psicológico para emergências em aviação: a teoria revista na prática
}

\author{
Maria Helena Pereira Franco \\ Pontifícia Universidade Católica de São Paulo
}

\begin{abstract}
Resumo
O presente trabalho aborda a experiência de atendimento psicológico em situações de emergência, em conseqüência de desastre aéreo. Focaliza intervenção em situações de luto traumático. O relato baseia-se nas experiências de um grupo de psicólogos, que tem o objetivo de oferecer atendimento psicológico especializado para situações de crises, catástrofes, emergências e luto, nos diferentes âmbitos de necessidade. Visa uma ação preventiva para situações de stress pós-traumático e luto traumático, bem como desenvolver habilidades nos profissionais envolvidos com essa atividade, de maneira a terem uma atuação eficiente, com risco controlado para sua saúde mental.
\end{abstract}

Palavras-chave: luto traumático; emergências; desastre aéreo

\begin{abstract}
Psychological support in aviation emergencies. This paper reports the experience of a group of psychologists with air disasters. It deals with traumatic grief, taking into consideration differences among victims and bereaved people in critical conditions. The objective of the work is to offer psychological treatment specific for crisis situations, catastrophes, emergencies and grief, for different needs of the victims, aiming at a preventive action for post-traumatic stress disorder and traumatic grief, and to develop abilities in the professionals that work with such activity, so that they provide an effective action, with controlled risk for their mental health.
\end{abstract}

Keywords: traumatic grief; aviation emergencies; air disaster

$\mathrm{E}$ m 1996, um desastre aéreo em São Paulo causou a morte de 99 pessoas, entre passageiros, tripulantes e um morador das casas atingidas. Um grupo de psicólogos atendeu familiares dos passageiros, funcionários da empresa aérea e moradores das ruas atingidas, a partir da experiência clínica já obtida com pessoas enlutadas. $\mathrm{O}$ atendimento se deu de maneira pontual, nos dias imediatos ao desastre, e também com contornos clínicos tradicionais, ao longo de meses após o mesmo (Falco, 2003) para as pessoas que apresentaram condições de risco para luto complicado (Doka, 1996). A experiência levou o grupo a profundas reflexões sobre esta modalidade de atendimento e gerou reformulações e desenvolvimentos teóricos que se revelariam de grande importância em situações futuras (Franco, 2003a).

Posteriormente, a partir de 1998, formou-se um outro grupo de psicólogos, especificamente com o objetivo de preparar-se para atuar em situações de emergência, relacionadas a desastres, traumas e luto traumático (Franco, 2003b). A proposta deste grupo, para promover "sobrevivência grupal", como recomendam Williams, Zinner e Ellis (1999, p. 6), Cohen
(2000) e American Psychiatric Association (1995), é: (a) oferecer atendimento psicológico especializado para situações de crises, catástrofes, emergências e luto, nos diferentes âmbitos de necessidade dos envolvidos, visando uma ação preventiva para situações de stress pós-traumático e luto traumático; (b) desenvolver habilidades nos profissionais envolvidos com essa atividade, de maneira a terem uma atuação eficiente, com risco controlado para sua saúde mental.

Este artigo focaliza o eixo luto-trauma, ampliando para as possibilidades de intervenção em situações de luto traumático, em consequiência de desastres aéreos. Assenta-se na dialética experiência-abstração (Stein, 2002b), de modo a entender e oferecer ajuda diante de um desastre. Comenta sobre a atuação deste grupo de psicólogos com experiência em atendimento a emergências, a partir de três desastres aéreos, guardadas as considerações sobre a inexistência de dois desastres iguais, o que leva, necessariamente, à flexibilidade na atuação, mesmo que seja pautada em um protocolo.

Para fazer parte deste grupo, a experiência mostrou as condições necessárias: (a) disponibilidade para ser acionado por 24 horas, 365 dias por ano, para ser contatado e 
posicionado em menos de 10 minutos; (b) disponibilidade para viajar para qualquer lugar do mundo; (c) domínio de, no mínimo, mais um idioma, além do Português; (d) treinamento para atendimento em situações de luto e participação nos treinamentos mensais específicos deste grupo, com número máximo de duas faltas permitidas por ano. Uma exigência absoluta está em assumir compromisso de sigilo sobre as atuações, incluindo nos contatos com a mídia. Presentemente, há 22 psicólogos no grupo, sendo que seis com doutorado em Psicologia Clínica, seis com mestrado (um cursando Doutorado), cinco cursando mestrado. Destes, nove já participaram de atendimento a acidentes aéreos.

$\mathrm{O}$ trabalho deste grupo responde diretamente às determinações do Departamento de Aviação Civil - DAC sobre assistência às famílias de passageiros vitimados em desastres aéreos.

A postura atual recomenda que a resposta ao desastre, com cuidados em situações traumáticas, se destine a sobreviventes machucados ou não machucados; parentes e amigos enlutados e traumatizados; equipe de assistência emergencial; membros da equipe de resgate e outros serviços de apoio; membros da mídia que cobriram o fato; e vítimas secundárias (Figley, Bride, \& Mazza, 1997; Hodkinson \& Stewart, 1998; Lewis, 1994; Stein, 2002a; Young, 1998). Como se observa, é amplo o espectro de pessoas atingidas por um desastre aéreo, mesmo que o órgão governamental brasileiro regulamente prestação de assistência exclusivamente às famílias.

Algumas definições se fazem necessárias, de acordo com Lewis (1994), James e Gilliland (2001). Entende-se crise como a interrupção em um estado previamente normal de funcionamento, que resulta em instabilidade e significativo desequilíbrio no sistema. Sua sintomatologia é complicada, abrange indivíduo, familiares e comunidade, sem que precise estar exclusivamente associada ao evento gerador. Ninguém fica imune ao impacto de uma crise, mas cada pessoa a enfrentará com seus recursos, mesmo que em circunstâncias semelhantes. Um trauma é definido como uma ruptura no tecido vivo, causado por um agente externo, como resultado de uma cirurgia, um ato violento, um desastre. Geralmente leva a um estado de crise. Pode ser também definido como um período de desequilíbrio psicológico, resultante de um evento ou situação danosa, assim constituindo um problema significativo que não pode ser resolvido com as estratégias de enfrentamento conhecidas. Uma experiência traumática se dá quando uma pessoa se confronta com a morte, ameaça de morte, ferimentos sérios em si ou no outro e reações de intensa dor, desamparo ou horror.

Desastres diferem de outros tipos de trauma, com relação à sua escala e efeitos, o que vai implicar um apoio diferenciado daquele oferecido em situações de perda e stress.

\section{Trauma psicológico em resposta a desastres}

As reações típicas ao trauma se apresentam como intensa angústia diante de situações que lembrem o momento traumático, ou mesmo algum aspecto referente a ele; reação fisiológica diante desta exposição: ansiedade, sensações físicas, sensação de pânico; diminuição do interesse e participação nas atividades rotineiras; sensação de estranhamento diante das outras pessoas, retraimento e isolamento; inabilidade para fazer projetos e medo de morrer (Hodgkinson \& Stewart, 1998).

O acontecimento traumático é persistentemente revivido em, ao menos, uma das seguintes maneiras (American Psychiatric Association, 1994): memórias recorrentes ou intrusivas, incluindo imagens, percepções e sonhos; agir ou sentir como se o evento traumático estivesse ocorrendo novamente, incluindo a sensação de reviver a experiência, ilusões, alucinações e flashbacks dissociativos; agir ou sentir como se a pessoa falecida estivesse viva e presente (incluindo alucinações). Outras reações ao trauma incluem evitação de eventos associados a ele, como indicado em, pelo menos, três dos seguintes critérios: esforço para evitar pensamentos, sentimentos ou conversas associadas ao trauma; esforço para evitar atividades, lugares ou pessoas que lembrem a pessoa perdida ou o próprio evento traumático; inabilidade em lembrar algum aspecto importante, relacionado à negação da perda; inabilidade para sentir e apegar-se.

Os sintomas do trauma incluem distúrbios do sono; irritabilidade, hostilidade em relação aos outros e explosões de raiva; dificuldade de concentração, compreensão e confusão; hipervigilância, desconfiança, agitação e insegurança; respostas exageradas aos estímulos do ambiente; suor excessivo, palidez, taquicardia, dor de cabeça, febre, desmaios e enjôo.

Em função do fato gerador do trauma, associado às demais condições que o definem, ele pode se manifestar pela Reação Aguda de Stress - RAS (aparecimento dos sintomas logo após o desastre e pela elaboração desses sintomas num período de um mês) ou pelo Distúrbio de Stress Pós-Traumático - DSPT, ou PTSD (persistência dos sintomas descritos anteriormente por mais de um mês ou aparecimento dos sintomas pelo menos um mês após o trauma). A descrição destas condições foi feita pela primeira vez pela American Psychiatric Association (1994), tendo sido retomada por Lewis (1994), Classen, Koopman, Hales e Spiegel (1998), Harvey e Bryant (1998) e Hodgkinson e Stewart (1998).

Estudos apontam que $75 \%$ das pessoas expostas a uma situação traumática necessitam ser adequadamente avaliadas quanto à possibilidade de apresentarem distúrbios psíquicos, com as complicações associadas: depressão, ansiedade e fobia, abuso de drogas e álcool (Freedy, Saladin, Kilpatrick, Resnick e Saunders, 1994; Giel, 1990; Green, 1994).

Como as reações a um desastre são muito variáveis, não é possível prever o tempo de que as pessoas traumatizadas necessitam para se recuperar. Há alguns fatores que podem contribuir ou impedir a recuperação destas pessoas, como a existência de sistemas de apoio dentro e fora da comunidade envolvida, o que ressalta a importância de apoio psicológico especificamente dirigido a estas necessidades. 


\section{O luto nas emergências}

A outra vertente para a compreensão da vivência da pessoa envolvida em um desastre é o luto, aqui entendido como reação normal e esperada para o rompimento de vínculo (Parkes, 1998). Tem como função proporcionar a reconstrução de recursos e viabilizar um processo de adaptação às mudanças ocorridas em conseqüência das perdas (Bromberg, 1995; Franco, 2002; Parkes, 1998). É um processo determinado por fatores tanto internos (estrutura psíquica do enlutado; tipo de vínculo com a pessoa falecida; histórico de perdas anteriores) como externos (circunstâncias da perda; crenças culturais e religiosas; apoio recebido).

A compreensão do luto como um processo permite entendê-lo ao longo de fases, das quais a primeira é a que mais toca de perto a realidade do atendimento em desastres aéreos. É a fase de entorpecimento, na qual a reação encontrada é de choque e descrença. O enlutado tem dificuldade em entrar em contato com a nova realidade e esta dificuldade é acentuada em situações de perda repentina ou inesperada. As fases seguintes (anseio e protesto, desespero e recuperação e restituição) podem se intercalar e têm duração variada.

Quando as reações perante as perdas não são as esperadas, isto é, fogem da sintomatologia e do processo descrito acima, é encontrado um processo de luto complicado.

Há fatores de risco para a instalação do luto complicado, entre os quais encontram-se aqueles relativos às circunstâncias da perda: mortes repentinas, violentas, consideradas prematuras pelo enlutado; a causa da morte e seu significado; o tipo da morte, destacando-se exposição à mídia, mortes estigmatizadas ou causadoras de vergonha ao ambiente social; existência de segredos relativos à morte ou à sua causa; falta de rituais; falta de suporte; outras perdas concomitantes a morte.

\section{Respondendo ao desastre}

James e Gilliland (2001) fizeram uma importante revisão acerca das abordagens utilizadas na compreensão e na atuação em situações de crise e afirmam que intervenção em crise é diferente de psicoterapia do luto e de psicoterapia focada no problema, ressaltando a importância de fatores sociais, desenvolvimentais, psicológicos, ambientais e situacionais que fazem com que um dado evento seja vivenciado como uma crise. Recomendam que um trabalho de intervenção em crise, como o atendimento psicológico em emergências, deve utilizar-se uma abordagem focal, embora problemas concomitantes sejam reconhecidos como importantes na dinâmica da situação-problema. O objetivo não é a modificação de características peculiares da pessoa em crise ou de seu padrão de personalidade. Portanto, é necessário perceber a configuração da situação de crise, sempre levando em conta as condições individuais, porque a intervenção deve contemplar ambos os aspectos, o genérico e o específico, fazendo uso de técnicas que considerem essa demanda (Hodgkinson \& Stewart, 1998). Estes princí- pios norteiam a ação deste grupo, uma vez que correspondem não somente aos aspectos técnicos utilizados, mas, sobretudo, a uma postura ética e epistemológica necessária para uma definição de protocolo de ação.

Nosso trabalho busca cuidar da intervenção em crise considerando diferentes necessidades, específicas às fases de atendimento (Hodgkinson \& Stewart, 1998; Nurmi, 1998): impacto, retração ou recuo, período pós-traumático. Também seguimos a orientação do grupo NOVA - National Organization for Victims Assistance (Young, 1998) sobre a seqüência no atendimento a vítimas de desastres aéreos, mesmo considerando que muitas dessas ações se sobrepõem e não é sempre possível estabelecer limites cronológicos ou seqüenciais rígidos diante das especificidades do desastre. Nossa atuação se apresenta, então, no $2^{\underline{0}}$ momento, de intervenção psicológica em emergência (intervenção em crise), antecedido do resgate físico e seguido de psicoterapia ou aconselhamento.

\section{Lidando com pessoas enlutadas e traumatizadas}

A pessoa enlutada em condições traumáticas está fragilizada e precisa de acolhimento, paciência e atenção; geralmente está desorganizada, incoerente, assustada, paralisada. Levando em conta estas condições peculiares, alguns cuidados são primordiais, na atitude em relação a esta pessoa. O que norteia nossa prática é o cuidado para não fazer com que a pessoa pare de sofrer rapidamente, pois isto seria um mecanismo de tamponamento de sua reação, com graves conseqüências. Assim sendo, cuidamos para não evitar o assunto e não desviar a conversa do tema.

A intervenção psicológica em emergência, como proposta por Young (1998) e realizada por este grupo, procura reduzir o stress agudo, causado pelo impacto do trauma, por meio de: (a) restaurar a dominância do funcionamento cognitivo sobre reações emocionais; (b) facilitar a restauração do funcionamento das instituições sociais e da comunidade; (c) facilitar o reconhecimento cognitivo do que aconteceu.

A intervenção psicológica em emergência procura também restaurar ou aumentar as capacidades adaptativas, por meio de: (a) oferecer oportunidades para as vítimas avaliarem e utilizarem apoio familiar ou da comunidade; oferecer educação sobre expectativas futuras e (b) oferecer oportunidade para os sobreviventes organizarem e interpretarem cognitivamente - o evento traumático.

\section{Lidando com perdas pessoais}

O profissional que atua exposto a situações de stress, como o que atende vítimas de desastres aéreos, apresenta também reações que podem ser, segundo Lewis (1994) e Hodgkinson e Stewart (1998): emocionais, físicas e cognitivas.

Isto requer, portanto, que junto a um profundo conhecimento das técnicas empregadas, o profissional tenha consciência de aspectos relevantes de sua condição pessoal para este tipo de atividade e possa identificar suas necessidades de descanso, alívio, até mesmo de afastamento da atividade. É um indivíduo em risco. 
Nos treinamentos do grupo, a distinção entre atendimento psicológico em emergência e psicoterapia é muito clara, embora as situações críticas apresentem uma dinâmica de tal ordem que pode ser considerado adequado e necessário que se fizesse uma intervenção com uso de recursos advindos de ambas as abordagens.

\section{Referências}

American Psychiatric Association. (1994). Diagnostic and statistical manual of mental disorders ( $4^{\mathrm{a}}$ ed.). Washington, DC: Autor.

American Psychiatry Association. (1995). Psychiatric dimensions of disaster: patient care, community consultation, and preventive medicine. Harvard Review of Psychiatry, 3(4), 196-209.

Bromberg, M. H. P. F. (1995). A psicoterapia em situações de perdas e luto. Campinas: Psy II.

Classen, C., Koopan, C., Hales, R., \& Spiegel, D. (1998). Acute stress disorder as a predictor of posttraumatic stress symptoms. American Journal of Psychiatry, 155(35), 620-624.

Cohen, R. E. (2000). Mental health services in disasters, manual for humanitarian workers. Cidade do México: El Manual Moderno \& Pan American Health Organization.

Doka, K. J. (1996). Living with grief after sudden loss: suicide, homicide, accident, heart attack, strok. Washington, DC: Hospice Foundation of America.

Falco, L. F. (2003). Vencendo a turbulência. In Rosa, M. (Org.), A era do escândalo, lições, relatos e bastidores de quem viveu as grandes crises de imagem (pp. 33-68). São Paulo: Geração Editorial.

Figley, C. R., Bride, B. E., \& Mazza, N. (Orgs.). (1997). The traumatology of grieving. Washington, DC: Taylor \& Francis.

Franco, M. H. P. (2002). Estudos avançados sobre o luto. Campinas: Livro Pleno.

Franco, M. H. P. (2003a). Psychosocial support in disasters as part of crisis intervention. Anais do $12^{\circ}$ Seminário Internacional de Psicologia da Aviação (pp. 403-405). Dayton: Ohio State University.

Franco, M. H. P. (2003b). Treinamento de psicólogos para atuar em emergências aeronáuticas. Anais do $2^{0}$ Seminário Brasileiro de Fatores Humanos em Ambiente de Aviação (pp.10-11). Rio de Janeiro: NUICAF.
Freedy, J. R., Saladin, M. E., Kilpatrick, D. G., Resnick, H. S., \& Saunders, B. E. (1994). Understanding acute psychological distress following natural disaster. Journal of Traumatic Stress, 7, 257-274.

Giel, R. (1990). Psychosocial process in disasters. International Journal of Mental Health, 19, 7-20.

Green, B. L. (1994). Traumatic stress and disaster: mental health effects and factors influencing adaptation. In F. Liehmac \& C. Nadelson (Orgs.), International Review of Psychiatry (vol. 2). Washington, D.C.: American Psychiatric Press.

Harvey, A., \& Bryant, R. (1998). The relationship between acute stress disorder and posttraumatic stress disorder. A prospective evaluation of motor vehicle accident survivors. Journal of Consulting and Clinical Psychology, 66(3), 507-512.

Hodgkinson, P. E., \& Stewart, M. (1998). Coping with catastrophe; a handbook of post-disaster psychosocial aftercare. Londres: Routledge.

James, R. K., \& Gilliland, B. E. (2001). Crisis intervention strategies. Londres: Brookes Cole.

Lewis, G. W. (1994). Critical incident stress and trauma in the workplace; recognition, response, recovery. Levinton: Accelerated Development.

Nurmi, L. (1998). The Estonian disaster: national interventions, outcomes, and personal impacts. In E. S. Zinner \& M. B. Williams (Orgs.), When a community weeps; case studies in group survivorship (pp. 49-71). Philadelphia: Brunner/Mazel.

Parkes, C. M. (1998). Luto, estudos sobre a perda na vida adulta. São Paulo: Summus.

Rosa, M. (2003). A era do escândalo, lições, relatos e bastidores de quem viveu as grandes crises de imagem. São Paulo: Geração Editorial.

Stein, H. F. (2002a). The December 3, 1999, fire in Worcester, Massachusetts, and the comparative study of disaster. Illness, Crisis \& Loss, 10(2), 164-173.

Stein, H. F. (2002b). Toward an applied anthropology of disaster: learning from disasters - experience, method, and theory. Illness, Crisis \& Loss, 10(2), 154-163.

Young, M.A. (1998). The community crisis response team training manual ( $2^{\underline{a}}$ ed.). Washington, DC: National Organization for Victims Assistance-NOVA.

Williams, M. B., Zinner, E. S., \& Ellis, R. R. (1999). The connection between grief and trauma: an overview. In E. S. Zinner \& M. B. Williams (Orgs.), When a community weeps; case studies in group survivorship (pp. 3-17). Philadelphia: Brunner/Mazel.

Maria Helena Pereira Franco, doutora em Psicologia Clínica pela Pontifícia Universidade Católica de São Paulo, é professora titular na mesma universidade e atua no Quatro Estações Instituto de Psicologia. Endereço para correspondência: Alameda Campinas, 1493, apto. 102; São Paulo, SP; CEP: 01404-002. E-mail: bromberg@pucsp.br 